\title{
PARADOJAS EPISTÉMICAS EN LA APROXIMACIÓN INFORMACIONAL A LA FÍSICA TÉRMICA
}

\author{
Epistemic Paradoxes in the informational approaches to Thermal Physics
}

\author{
JAVIER ANTA \\ Universidad de Barcelona - Logos, SPAIN \\ antajav@gmail.com
}

\begin{abstract}
In this paper I aim to assess the conceptual and interpretative basis of the infothermal paradox' underlying informational approaches to thermal physics (particularly the work of Jaynes [1957] and Brillouin [1962]), claiming that information both increases and decreases when observing a system approaching thermal equilibrium. I defend that, even when different concepts and types of information are distinguished, the paradox persist because of a robust connection (epistemic, informational and theoretical) between the information-increasing and the information-decreasing side of this thesis. Finally, I will conclude by arguing that the info-thermal paradox properly manifests deep conceptual-interpretative inconsistencies underlying information-centric thermal physics.
\end{abstract}

Keywords: Information Physics • Jaynes • conceptual paradoxes

ACCEPTED: 09/09/2020

The articulation of our physical knowledge is one of the chief tasks of philosophy of physics. Much of the work is interpretative in nature.

Belot 1999

\section{Introducción}

Existe una significativa tendencia actual dentro de las ciencias naturales que se basa en comprender distintos fenómenos físicos a partir del concepto de información. Esta se remonta al menos a la popularización de la teoría matemática de la información Shannon dentro de la termofísica durante los años cincuenta, principalmente a manos del "Science and Information Theory" (1961) de Brillouin, y Jaynes (1957) con su "Information Theory and Statistical Mechanics". En el primer caso se postula que la entropía de un sistema y la información que se tiene acerca del sistema son medidas complementarias; en el segundo, que la entropía y la información son conceptos 
idénticos. Sin embargo, la medida técnica de información que propuso Shannon presentaba ciertos problemas conceptuales a la hora de aplicarse a ciertos contextos de práctica científica, por lo que Brillouin desarrollará una alternativa a esta:

Brillouin concept was designed to overcome an anomaly in Shannon's theory: the more random is the arrangement of symbols - ie, the higher the entropy - the greater the information content. Taken to its logical extreme this would means that pure noise, which contains the greater amount of entropy, would contain the greatest amount of information. Shannon and Weaver were aware of this problem. (Stonier 1990)

El contenido de esta cita muestra con claridad lo que denominaremos como 'paradoja info-térmica', la cual representa de manera significativa las profundas inconsistencias conceptuales de la aproximación informacionalista a la termofísica: con respecto a un gas que se aproxima hacia su equilibrio térmico, la información que un observador posee acerca de la configuración molecular del gas incrementa y disminuye al mismo tiempo. El objetivo principal de este artículo es evaluar los fundamentos conceptuales e interpretativos que dan lugar a esta paradoja.

El plan para este artículo es el siguiente: en primer lugar, presentaremos los elementos básicos del formalismo mecánico estadístico de Boltzmann y Gibbs (Callender 1999), en tanto que constituyen los cimientos teóricos sobre los que se cimentan los principales paradigmas informacionalistas: los basados en el principio de Neguentropía de Brillouin (1962) y el principio de Máxima Entropía de Jaynes (1957), respectivamente. Nuestra segunda sección estará dedicada a delimitar el contenido de la 'paradoja info-térmica', la cual sentencia que en el caso de un gas que se aproxima al equilibrio, la información que posee un observador con respecto al gas incrementa y disminuye simultáneamente. Posteriormente procederemos a explicar las causas conceptuales-interpretativas de dicha paradoja, principalmente (a) la confusión entre información de Shannon (1948) y el sentido ordinario de información y (b) la adopción de interpretaciones informacionales laxas e injustificadas de la entropía (Lewis 1930, Shannon \& Weaver 1949). Para concluir, evaluaremos como la paradoja info-térmica emerge en casos concretos de análisis informacionales de procesos térmicos, como el llevado a cabo por Ben-Naim (2008), mostrando como las inconsistencias conceptuales-interpretativas que soportan la paradoja info-térmica conllevan deficiencias epistémicas decisivas.

\section{Mecánica estadística y paradigmas informacionalistas}

El informacionalismo, entendido este como la tesis por la que la información (definida de un modo u otro) juega un papel clave en la comprensión de nuestra realidad, posee una historia intrincada como corriente de pensamiento científico dentro de la 
física. En particular, fue durante los años cincuenta del siglo veinte cuando comenzaron a emplearse de forma explícita y sistemática conceptos informales, sobre todo a raíz de la recién publicada teoría matemática de la comunicación de Shannon (1948) (posteriormente popularizada como 'teoría de la información'). Es durante esta década cuando surgieron dos de las principales tradiciones físico-informacionalistas que llegan hasta nuestros días ${ }^{1}$, a saber: el jaynesianismo (Jaynes 1957), el cual reinterpreta epistémicamente la mecánica estadística a partir del lema 'información y entropía son equivalentes'; y el brillouinismo (ver Brillouin 1961), erigido sobre una interpretación complementarista de la información como "entropía negativa". Ambas perspectivas se asientan teóricamente sobre los formalismos mecánico estadísticos de Gibbs (1902) y Boltzmann (1896), respectivamente, por lo que procedemos a presentarlos brevemente a continuación.

\subsection{Mecánica estadística de Gibbs e interpretación teórico informacional de Jaynes}

Para comprender en profundidad el jaynesianismo, antes debemos delinear el andamiaje teórico que presupone la aproximación gibbseana a la termofísica estadística. Gibbs (1902) formuló su aproximación a la mecánica estadística en base a lo que se conoce como 'ensamblajes estadísticos', los cuales que no son sino copias virtuales de los microestados de un único sistema físico, usualmente gases u otros sistemas moleculares, representadas estadísticamente dichas 'copias virtuales' mediante una distribución de probabilidad $\varrho$ sobre el espacio de fase $\Gamma$. Con 'microestados' nos referimos a descripciones cuantitativas de la configuración microscópica de un sistema molecular (determinando la posición y velocidad de todas las moléculas que conforman el gas) y $\Gamma$ es un espacio formal $6 \mathrm{~N}$-dimensional que codifica datos posicionales y velocidades moleculares, donde $\mathrm{N}$ es el número de moléculas del sistema en cuestión. Curiosamente, la evolución temporal de $\varrho$ se asemeja al movimiento de un fluido incompresible para sistemas hamiltonianos. Desde esta perspectiva gibbseana, los valores de fase promedio de $\varrho$ son utilizados para codificar propiedades termodinámicas (macroscópicas o macroestadísticas), salvo para el caso particular de la entropía:

$$
\text { 1. } \mathrm{S}_{\mathrm{G}}[\varrho]=-k \int_{\Gamma} \varrho(x) \log \varrho(x) \mathrm{d} \Gamma
$$

en la que se integra sobre el volumen fásico $\mathrm{d} \Gamma$ correspondiente a los microestados $x$ compatibles con $\varrho$, todo multiplicado por $k$, la constante de Boltzmann. Esta cantidad entrópica, también llamada 'entropía de ensamblajes', alcanza su valor máximo cuando el sistema se encuentra el equilibrio térmico. Para aquellos sistemas cinéticos o simplemente 'gases' en equilibrio térmico sus posibles estados se representan 
estadísticamente mediante un 'ensamblaje' o 'distribución canónica' $\varrho$ (en la que la temperatura $\mathrm{T}$, el volumen $\mathrm{V}$ y el número de moléculas $\mathrm{N}$ son parámetros constantes), lo cual facilita enormemente el cálculo probabilístico de datos microestadísticos. En este último escenario teórico altamente ideal, la entropía gibbseana (1) torna aproximadamente equivalente a la entropía termodinámica salvo por una constante aditiva (Callender 1999).

Sobre tales cimientos teórico-formales se erige el jaynesianismo, el cual se basa en interpretar las probabilidades codificadas en $\varrho$ como cantidad de 'incertidumbre predictiva' (Hilgevoord y Uffink 1991) que posee un observador con respecto a la configuración molecular-microscópica de un sistema molecular en función del conocimiento que se tiene sobre sus cantidades termodinámico-macroscópicas observables. El conocido como 'Principio de Máxima Entropía' de Edwin Jaynes (1957a), núcleo teórico de su aproximación información-teórica a la mecánica estadística, consiste en un algoritmo que genera nuevas probabilidades $\varrho$ ' sobre los microestados $x$ de manera que se maximiza la predictibilidad estadística y la entropía de Shannon (ver Parker 2011, p.836) codificada en tal distribución:

1. $\mathrm{S}_{\mathrm{I}}[\varrho]=-\int_{\Gamma} \varrho(x) \log \varrho(x) d x$

Nótese que esta medida entrópico-informacional de Shannon (definida sobre los valores continuos de $\Gamma$ ) es formalmente similar a la entropía gibbseana (1), salvo por la constante de Boltzmann $k$ en el caso de la segunda. Sin embargo, desde la perspectiva informacional de Jaynes, estas no solo son formalmente similares (Frigg \& Werndl 2013, p.129), sino conceptualmente idénticas ${ }^{2}$, lo cual permite usar teóricamente el concepto de Shannon para predecir los valores precisos que tomarán las propiedades macroestadísticas observables de un sistema una vez se maximiza la cantidad (2) en la nueva distribución $\varrho$ '. Estas predicciones informacionales de valores macroscópicos se llevan a cabo en base a los promedios de fase de $\varrho$ 'a lo largo de su evolución temporal, de acuerdo con el algoritmo generador de inferencias derivable desde el Principio de Máxima Entropía. En este sentido, la mecánica estadística à la Jaynes no constituye una teoría referida directamente al comportamiento térmico de ciertos sistemas cinéticos, sino propiamente una teoría acerca de los recursos epistémicos e inferenciales con los que cuenta cualquier observador para determinar la configuración microscópica de tales sistemas-modelos.

\subsection{Mecánica estadística de Boltzmann y el principio neguentrópico de Brillouin}

Así mismo para comprender los fundamentos teóricos del brillouinismo cabría presentar el formalismo mecánico estadístico boltzmanniano. Boltzmann introdujo cier- 
tos postulados probabilísticos para formalizar matemáticamente cómo ciertas propiedades termodinámicas se derivarían a partir del comportamiento dinámico de las moléculas que componen dichos sistemas. En primer lugar, el espacio de fase $\Gamma$ se particionaría (o 'coarse-graining') en un conjunto de regiones fásicas que no se solapan y cuya unión topológica cubre $\Gamma$ completamente, en donde cada región corresponde a un macroestado $\mathrm{M}_{\mathrm{i}}$ que representa a su vez el valor de una determinada cantidad macroscópica observable (por ejemplo, temperatura, presión o volumen). En este sentido cada macroestado $\mathrm{M}_{\mathrm{i}}$ se compone de un número (infinito, en tanto que asumimos que $\Gamma$ es continuo) o volumen de microestados $x$ compatibles o indistinguibles macroscópicamente. A partir de estos elementos teóricos podemos definir la entropía de Boltzmann $\mathrm{S}_{\mathrm{B}}$ de un microestado particular $x$ (a diferencia de la entropía gibbseana, definida sobre distribuciones de probabilidad $\varrho$ ) como el volumen de fase del macroestado M que contiene $x$ :

1. $\mathrm{S}_{\mathrm{B}}=\mathrm{k} \log \left|\Gamma_{\mathrm{M}}\right|$

Desde una perspectiva boltzmanniana, el estado de equilibrio térmico corresponde al macroestado $\mathrm{M}^{*}$ con mayor volumen fásico de todo $\Gamma$. De hecho, $\mathrm{M}^{*}$ ocupa la práctica totalidad del espacio de fase para sistemas macroscópicos, en tanto que casi todas las posibles configuraciones moleculares (macroscópicamente indistinguibles) un sistema molecular pertenece a su macroestado de equilibrio térmico (Callender 1999, p.356). Por ello, en tanto que asumimos que la probabilidad de un microestado $x$ es proporcional al volumen del macroestado $\mathrm{M}$ en el que se encuentra $x$, el estado macroscópico más probable en el que se encontrará cualquier gas es el de equilibrio térmico; mientras que es altamente improbable que todas las moléculas de un gas se encuentren localizadas en un ínfimo volumen del contenedor del gas. Otra de las premisas probabilísticas clave es que la evolución microestadística $x$ del sistema recorrerá macroestados exponencialmente más volumétricos hasta alcanzar la región fásica correspondiente al equilibrio térmico (aunque las fluctuaciones térmicas son teóricamente posibles), lo cual no es sino una reinterpretación estadística de la segunda ley de la termodinámica.

A partir del formalismo mecánico estadístico que acabamos de dibujar, el físico franco-americano Leon Brillouin (1962) (uno de los principales adalides del uso de nociones informacionales dentro en el campo de la física térmica) desarrolló el conocido como 'principio de neguentropía', núcleo de su propuesta informacionalista. Este establece que la información (no nos referimos aquí a la noción de Shannon, tal y como veremos adelante) acerca de la configuración microscópica actual de un modelo cinético es inversamente proporcional al número de complexiones de Plank o volumen fásico del macroestado particular $\mathrm{M}_{\mathrm{i}}$ en el que se encuentra su microestado $x$. Es decir, mientras menos microestados compatibles (o equivalentemente menos 
volumen fásico) posea el macroestado $\mathrm{M}_{\mathrm{i}}$ que describe estadísticamente el modelo, menos 'incertidumbre inferencial' (Hilgevoord y Uffink 1991) poseerá un observador acerca de la configuración molecular actual del modelo cinético en cuestión. Por tanto, el principio de neguentropía establece una relación inversamente proporcional entre 'información' brillouiniana $I$ (codificada en complexiones de Plank W) y entropía boltzmanniana negativa -S. Cabe remarcar así mismo que esta tesis descansa sobre una concepción de la entropía como medida, no ya del desorden molecular o microscópico que encontramos en una sustancia, sino de la información requerida para describir la estructura microscópica de un sistema físico (Brillouin 1962, p.166). Esta interpretación neguentrópica de la noción de información desarrollada por el franco-americano se inspira directamente en la interpretación del concepto de entropía como 'carencia de información sobre el microestado particular del sistema' llevado a cabo por primera vez por el químico G. Lewis (1930), y de la que trataremos en la Sección 5.2.

Como estrategia para evitar las críticas de trivialidad ${ }^{3}$, Brillouin acuñará la noción de 'información ligada' para distinguir entre cantidad información sin contenido físico (cantidad abstracta) y aquella medida informacional I con carácter físico-empírico, dónde esta última queda propiamente definida propiamente como la diferencia $S_{0}-$ $S$ entre la entropía actual de un sistema y la entropía del mismo sistema en equilibrio térmico. Por tanto, la información ligada mediría la proximidad de un sistema cinético a su estado de equilibrio térmico o estado de mínima información brillouiniana.

\section{Encuadrando la paradoja info-térmica}

Acabamos de exponer como dos de las principales tradiciones informacionalistas en física térmica durante la segunda mitad del siglo veinte, el paradigma jaynesiano y brillouiniano (levantados sobre los principios teóricos de Máxima Entropía y de Neguentropía, respectivamente), parten de dos maneras diferentes de comprender los fenómenos térmicos desde el compartimento mecánico de la materia: a saber, el formalismo mecánico-estadístico de Gibbs basado en ensamblajes estadísticos y el de Boltzmann centrados en sistemas particulares. En este sentido, ambas constituyen perspectivas aparentemente irreconciliables, no solo en cuanto a sus fundamentos físico-teóricos sino también informacionales. Por una parte, el jaynesianismo parte de la identificación conceptual entre la medida entrópica de Gibbs y la informacional de Shannon para dotar de significación física a la segunda a la hora de generar predicciones efectivas; por otra parte, Brillouin elaboró una medida particular de información inversamente proporcional a la cantidad de complexiones indistinguiblescompatibles con un sistema, imprescindibles para definir la entropía de Boltzmann.

Sería un error asumir que dichas tradiciones informacionalistas se mantendrán 
como compartimentos estancos durante sus sesenta años de desarrollo posterior. Por el contrario, estas evolucionarán históricamente de manera compleja, entrelazando sus aparatos conceptuales e integrando sus bases interpretativas ${ }^{4}$; en especial a partir de los años noventa, década en la que el informacionalismo se radicalizará y alcanzará una popularidad desbordante entre la comunidad científica (encontrándonos eslóganes famosísimos como el "It from bit" de Wheeler o el "Information is physical" de Landauer). Defenderé aquí que esta moda obstinada en la historia reciente del pensamiento físico viene indisociablemente acompañada de una falta absoluta de rigor crítico y analítico a la hora de evaluar las bases propiamente conceptuales e interpretativas sobre las que se asienta dicho movimiento. En este sentido nos alineamos filosóficamente tanto con la crítica de Norton (2005) a la termodinámica de la computación (rama de la física informacional que parte del principio de Landauer) como con el rechazo de Timpson (2013) a la interpretación sustancialista de la información cuántica.

Nuestro principal objetivo durante este artículo es analizar una de las consecuencias directas de la profunda inconsistencia conceptual-interpretativa del informacionalismo físico en la actualidad, lo que podemos bautizar telegráficamente como 'paradoja info-térmica'. Esta puede expresarse inicialmente del siguiente modo.

\section{(PIT-A) Paradoja Info-Térmica:}

Dado un sistema físico cuyas propiedades térmicas actúan de acuerdo a la segunda ley de la termodinámica, (i) el observador obtiene progresivamente más información acerca del sistema durante su evolución dinámica, y al mismo tiempo (ii) el observador posee cada vez menos información sobre dicho sistema.

Dicha tesis (formulada muy superficialmente en PIT-A) no es sino la conjunción en una única proposición de, por un lado, (i) la tesis jaynesiana que afirma que la información de un sistema aumenta a medida que se aproxima al equilibrio, y (ii) la idea brillouiniana de que la información sobre este sistema disminuye durante este mismo proceso. Recordemos que, al menos formalmente, una paradoja es la derivación de una contradicción, autorreferencia o circularidad viciosa a partir de una única proposición (auto-contradictoria) o de la conjunción de varias proposiciones, como es el caso de (i) 'la información del observador aumenta' y (ii) 'la información del observador disminuye' en PIT-A. En nuestro caso particular, la paradoja no emerge inmediatamente de forma lógica a partir del contenido de sus componentes conceptuales (e.g. la paradoja del mentiroso: "esta frase es falsa") sino de la interpretación particular que se les otorga a tales conceptos. Por ejemplo, el concepto mismo de 'información' empleado implícitamente en (i) es radicalmente diferente al que se utiliza en (ii). Reformulando PIT-A de un modo más técnico obtenemos y explícito obtenemos: 


\section{(PIT-B) Paradoja Info-Térmica:}

Dado un sistema físico cuya entropía termodinámica crece monotónicamente durante su evolución dinámica hasta llegar al estado de equilibrio térmico (de acuerdo con la segunda ley de la termodinámica) entonces: (i) el observador perderá información brillouiniana acerca de la posición y velocidad de sus componentes moleculares, en tanto que cada vez más complexiones se tornan macroscópicamente indistinguibles; y al mismo tiempo (ii) ese mismo observador poseerá cada vez más información shannoniana acerca de la distribución de probabilidad actual sobre las posiciones y velocidades moleculares de dicho sistema físico.

En esta nueva formulación se ve con claridad el grave problema conceptual que desentraña la paradoja info-térmica, no solo a nivel informacional sino también en los fundamentos teóricos mismos de la mecánica estadística en los que descansa. PITB posee dos partes distinguibles, por un lado (la que podemos denominar como su faceta 'brillouiniana') (i) el observador pierde información epistémica acerca del microestado fáctico del gas a medida que este avanza hacia el equilibrio, debido a que aumentan logarítmicamente las configuraciones moleculares macroestadísticamente indistinguibles. Por otra parte (ii) el observador incrementa enormemente la cantidad de información macroestadística que se puede llegar a predecir (de acuerdo al principio de Máxima Entropía expuesto en la Sección 2.1), mostrando así su también existente faceta jaynesiana. Ilustremos PIT-B con un modelo cinético particular como el ilustrado en la Figura 1, en la que el gas que encuentra inicialmente en un estado alejado del equilibrio térmico (baja entropía de Shannon, alta información de Brillouin) y evoluciona progresivamente hacia este (máxima entropía de Shannon, mínima información de Brillouin).

Nótese que a pesar de distinguir las diferentes descripciones (jaynesiana y brillouiniana) y conceptos informacionales (entropía información-teórica de Shannon e información-neguentropía de Brillouin, respectivamente) de PIT-A en PIT-B, el carácter paradójico de dicha afirmación simultanea no se disuelve en tanto que el objetivo epistémico es el mismo: obtener conocimiento acerca de la configuración molecular de un sistema cinético. El argumento que aquí pretendo defender no es que el brillouinismo y el jaynesianismo sean contradictorios, sino que la aplicación sistemática de aparatos informacionales heterogéneos en modelos termofísicos genera profundas inconsistencias conceptuales, como muestra la paradoja que aquí nos concierne. En lo que resta de este artículo analizaremos en detalle el origen conceptual e interpretativo de la persistencia de la paradoja al distinguir-delimitar las distintas descripciones informacionales y conceptos de información implicados en esta. Tal y como remarcaremos, la persistencia de la paradoja (de PIT-A a PIT-B) se hace evidente en cuanto reinterpretamos los conceptos mencionados desde una perspectiva epistémica.

Por un lado, recordemos que para la mecánica estadística de Jaynes, al identificar la entropía gibbseana $\mathrm{S}_{\mathrm{G}}$ con la medida informacional de Shannon $\mathrm{H}$, ambas 


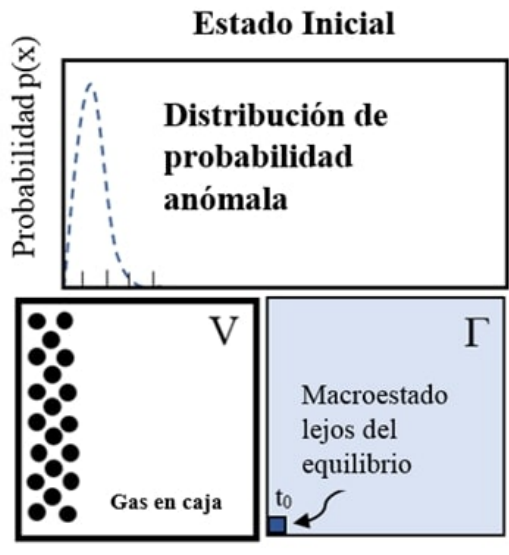

Fácil de distinguir

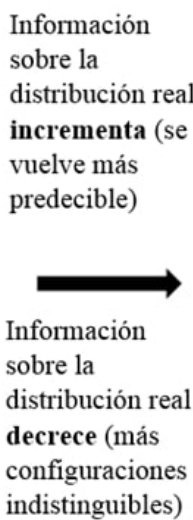

indistinguibles)

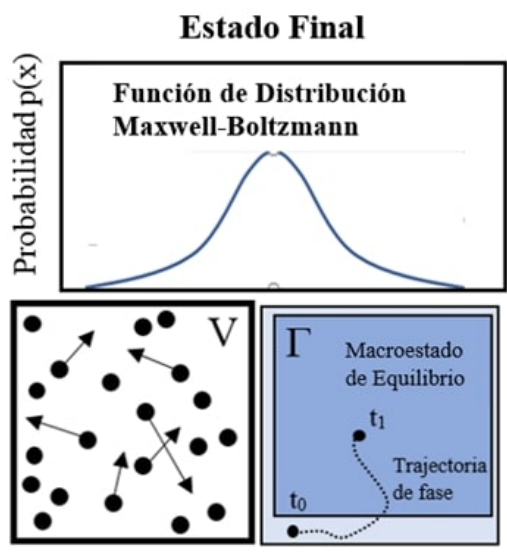

Difícil de distinguir

Figura 1: Paradoja info-térmica en un modelo cinético durante un proceso irreversible de expansión. (Triple izquierdo) El modelo cinético en encuentra inicialmente $t_{0}$ en un estado alejado del equilibrio térmico (gas confinado en un lado del contenedor V), por lo que se representa estadísticamente con una distribución probabilística anómala y cuyo microestado se haya contenido en un macroestado minúsculo en el espacio de fase $\Gamma$. (Triple derecho) Al final del proceso en $t_{1}$ el modelo alcanza su equilibrio térmico expandiéndose por $\mathrm{V}$, representado estadísticamente mediante la distribución de Maxwell-Boltzmann y alcanzando el macroestado de máximo volumen de fase en $\Gamma$.

alcanzarían su valor máximo (volviéndose máximamente predecible el valor de sus cantidades observables) cuando el sistema físico alcanza su equilibrio térmico. La interpretación epistémica de la medida información-teórica de Shannon como medida de predictibilidad queda claramente ilustrada a partir del procedimiento de etiquetación de Frigg ${ }^{5}$ (2004): dividiendo el espacio de fase $\Gamma$ en un conjunto de regiones discretas iguales $\Gamma_{R}$ a las que se le asigna un símbolo-etiqueta y cuya unión cubre topológicamente $\Gamma$, la evolución dinámica de un sistema que se encuentra ya en equilibrio térmico daría como resultado una secuencia de símbolos completamente aleatoria. Dicha secuencia simbólica aleatoria (entendida información-teóricamente como un 'mensaje') codificaría la máxima cantidad de información de Shannon H. Así mismo y desde una perspectiva jaynesiana, esta secuencia-mensaje representaría un estado epistémico de máxima predictibilidad, no con respecto a la ocurrencia de símbolos (puesto que esta es máximamente impredecible) sino acerca de la distribución de probabilidad: mientras más cerca se encuentra el sistema cinético de su equilibrio, más fácil es para un observador el predecir que sus valores microscópicos de distri- 
buyen de manera homogénea. Por tanto, todo sistema o modelo termo-cinético que haga válida a la segunda ley de la termodinámica incrementaría monotónicamente la cantidad de información de Shannon codificada en su distribución estadística $\varrho$ a medida que dicho ensamblaje evoluciona hacia el equilibrio térmico (representado por una distribución de probabilidad microcanónica), volviéndose en consecuencia más y más predecible.

Por otra parte, la noción informacional empleada en la descripción brillouiniana guarda una mayor relación con el concepto cotidiano de 'información' (Earman \& Norton 1999, p.8), con claro contenido semántico (información sobre el microestado actual del gas) y epistémico, que con la noción técnica de Shannon. Dicha noción epistémica de información queda formalmente codificada mediante complexiones de Planck W, cuya cantidad es inversamente proporcional al volumen de fase de un macroestado particular (información libre) o a la diferencia de volúmenes fásicos entre distintos macroestados correspondientes a distintos momentos en la evolución dinámico-estadística del sistema (información ligada). Tal y como hemos mencionado previamente en esta sección, a menor volumen fásico, menos microestados son compatibles con el estado actual del sistema y por tanto más información epistémica relevante o certeza (ratio entre microestado actual $x$ y microestados posibles $X$ ) tendremos acerca del mismo. En el este sentido, todo sistema-modelo cinético alcanzaría eventualmente su mínima cantidad de información cuando llega a su estado de equilibrio térmico, tras 'eliminar información' de manera espontánea durante su evolución temporal. En palabras de Brillouin "Increase of entropy and loss of information proceed together. We may say that the gas progressively "forgets" the information" (1962, p.157)

A partir de estas interpretaciones epistémicas de la entropía información-teorética Shannon como impredecibilidad macroestadística y la información neguentrópica de Brillouin como indistinguibilidad microestadística (también interpretables en términos de Uffink [1990] como incertidumbre predictiva e incertidumbre estadísticoinferencia, respectivamente) podemos reformular la paradoja info-térmica en términos más claros: nuestro conocimiento acerca de la configuración microscópica de un sistema (predecible macroestadísticamente y distinguible microestadísticamente) disminuye y aumenta a lo largo de su aproximación al equilibrio. Dicho de otro modo, el microestado determinista de un sistema es extremadamente difícil de distinguir y fácil de predecir (Figura 1) cuando este se encuentre en equilibrio térmico. Es decir:

\section{(PIT-C) Paradoja Info-Térmica:}

Dado un sistema físico cuya entropía termodinámica crece monotónicamente durante su evolución dinámica hasta llegar al estado de equilibrio térmico, entonces (i) el observador aumentará su incertidumbre acerca de la posición y velocidad de sus componentes moleculares, en tanto que la dificultad para distinguir su configuración 
molecular particular aumenta exponencialmente, y al mismo tiempo (ii) ese mismo observador tendrá muchos más recursos para predecir la distribución de probabilidad actual sobre las posiciones y velocidades moleculares de dicho sistema físico.

\section{Evaluando el puzle conceptual-interpretativo de la paradoja}

En la sección anterior hemos visto como los principios de Máxima Entropía de Jaynes y el principio de Neguentropía de Brillouin generan diferentes descripciones informacionalistas de procesos térmicos: la información de un observador con respecto a un gas aproximándose al equilibrio aumenta y disminuye al mismo tiempo (PIT-A). Hemos defendido que tras especificar los conceptos de información implícitos y los detalles técnicos particulares con (PIT-B), el carácter paradójico de ambas descripciones reside no en la incompatibilidad de ambas descripciones sino en la inconsistencia de sus conceptos, lo cual queda ilustrado mediante una reinterpretación epistémica de las tesis informacionales: el conocimiento de un observador respecto a la configuración molecular actual de un sistema aumenta y disminuye al mismo tiempo (PITC). En esta sección veremos como la paradoja no solo se origina-persiste al integrar perspectivas informacionales incompatibles (jaynesianismo y brillouinismo) o reconceptualizar epistémicamente sus respectivas descripciones, sino que por el contrario su fuente es mucho más compleja y abarca una pluralidad de frentes adicionales, a saber: (a) la confusión entre el concepto informacional de Shannon y la noción neguentrópica de Brillouin, y (b) el entrelazamiento de diferentes interpretaciones de la conexión entre medidas entrópicas e informacionales.

\subsection{Nociones de información y confusión conceptual}

Por un lado, es más que evidente que el término informacional que aparece en la faceta jaynesiana de la paradoja es la noción técnica de Shannon. Este se suele tomar paradigmáticamente como una medida de lo impredecible que es la transmisión de una secuencia de símbolos (o 'mensaje'), por lo que depende enteramente de la frecuencia de ocurrencia $\mathrm{p}\left(x_{\mathrm{i}}\right)$ de cada símbolo individual $x_{\mathrm{i}}$ codificada en la función de probabilidad distribuida sobre la fuente (o conjunto de símbolos que pueden conformar un mensaje). Por ejemplo, el mensaje 'XYZXW' contiene más información de Shannon que el mensaje 'HABLAR' si consideramos la frecuencia de las letras del inglés. En este sentido, el valor de la medida información-teórica $\mathrm{H}(X)$ de Shannon depende de lo improbable que son los símbolos y de ninguna manera del contenido o valor semántico de los símbolos que conforman el mensaje, por tanto, sería teóricamente impreciso interpretarlo como un concepto robustamente semántico ${ }^{6}$. La 
información shannoniana no es propiedad de un único mensaje o un único estado de cosas particular (a diferencia de la información cotidiana y brillouiniana), sino propiamente del mensaje-estado con respecto a la fuente y su distribución probabilística. De este modo la información estadística de Shannon se nos presenta alejada de nuestras intuiciones cotidianas acerca de lo que es la información y por tanto también de sus ventajas epistémicas a la hora de aplicarlo en contexto de práctica científica:

The concept of information developed in this theory at first seems disappointing and bizarre-disappointing because it has nothing to do with meaning, and bizarre because it deals not with a single message but rather with the statistical character of a whole ensemble of messages (Weaver 1949)

Dotar de carácter semántico a la información shannoniana (interpretarla como información sobre algo) en un contexto mecánico estadístico provoca una confusa conexión conceptual de esta con el contenido información semántico-epistémica de Brillouin, y en consecuencia da lugar a la paradoja info-térmica, tal y como veremos detenidamente en la Sección 5.2. Se debería defender que la viabilidad de una interpretación semántica robusta de la información de Shannon en un ámbito científico particular depende intrínseca y localmente de la arquitectura conceptual de tal ámbito, siendo la biología una de las arenas más prolíficas para dicha tarea (ver Marcos 2011). Además, si una interpretación semántica robusta y conceptualmente coherente de la información teórico-informacional fuese posible, entonces podríamos argumentar que en cada una de las facetas de la paradoja esta cantidad refiere a un sistema real diferente; sin embargo, aquí defenderemos que esto no es posible en mecánica estadística ${ }^{7}$. Timpson (2013, p.30) defiende también esta profunda separación conceptual entre información de Shannon y su versión cotidiana, ya que únicamente coincidirían en un escenario artificial en el que las probabilidades corresponden exactamente a los datos extraíbles en un experimento, el cual es absolutamente irrelevante en casos reales de prácticas científicas. ${ }^{8}$

Para defender su falta de carácter semántico deberíamos subrayar también que la medida informacional de Shannon fue concebida dentro de un contexto teórico muy particular. Shannon (1948) perfiló un modelo comunicativo matemático basado en el quíntuple emisor-codificador-canal-decodificador-receptor, en donde el teorema de codificación no-ruidosa de Shannon nos proporcionaría la forma óptima de comprimir-descomprimir y transmitir un mensaje (formalizado como un proceso aleatorio de Markov) a través de un canal con ruido. Entre otras, la noción de 'ruido' cuantifica la cantidad de información que llega al receptor y no proviene del emisor, así como la de 'equivocación' refiere a la información transmitida que no llega al receptor. Este es, a muy grandes rasgos, el contexto teórico concreto en el que se desarrolló $\mathrm{H}$. En este punto defenderé que, en tanto que la medida informacional de Shannon aparece implícitamente en la paradoja info-térmica únicamente en su 
conexión formal con la entropía estadístico mecánica de Gibbs (mediante la identificación de Jaynes), el modelo-formalismo comunicativo emisor-receptor no juega ningún papel teórico relevante en el contexto teórico jaynesiano.

Por otro lado, la cara brillouiana de nuestra paradoja depende teóricamente de su concepto de información, muy ligado a la noción ordinaria del mismo. A pesar de postular el principio de neguentropía (información como neguentropía) a partir de la tesis lewisiana (entropía como información negativa) como conexión teórica entre ambas cantidades, no cabe duda que su mayor innovación consiste en el ejercicio interpretativo llevado a cabo con respecto a ambos:

Entropy is usually described as measuring the amount of disorder in a physical system. A more precise statement is that entropy measures the lack of information about the actual structure of the system. This lack of information induces the possibility of a great variety of microscopically distinct structures, which we are unable to distinguish from one another. Since anyone of these different microstructures can actually be realized at any given time, the lack of information corresponds to actual disorder in the hidden degrees of freedom. (Brillouin 1962, p.160)

En primer lugar, la interpretación de la entropía térmica como medida del desorden existente en los componentes moleculares de una substancia se basa en el grado de regularidad estructural con el que se disponen microscópicamente dichos componentes. Esto lo mostró Boltzmann (1896) mediante los distintos estados físicos que pueden tomar una substancia, desde los más ordenados y mínimamente entrópicos (por ejemplo, sólidos cristalinos, cuyas moléculas se interconectan en parámetros regulares) hasta los más desordenados y altamente entrópicos, e.g. gases ideales, cuyos componentes apenas interactúan. Fue Schrödinger (1944) quien postuló en el contexto de la biología molecular que cristales altamente irregulares podrían codificar una mayor cantidad de 'datos' (aunque no mencionó explícitamente el término 'información'), conteniendo del mismo modo un menor grado de orden-regularidad molecular o entropía negativa. De este modo, la entropía física de un sistema no solo codificaría la falta de información de un observador (à la Lewis) con respecto a un sistema-modelo molecular, sino también la capacidad objetiva de un sistema físico de codificar datos microscópicamente.

Brillouin (1962) partirá de esta línea interpretativa para desarrollar (a partir de su principio de Neguentropía) una noción de información con evidente carácter semántico-epistémico, en tanto que depende del estatus epistémico de un observador con respecto al estado microscópico particular de un sistema molecular. De este modo el franco-americano solventa el problema teórico que presenta la aplicación de la noción informacional de Shannon en el ámbito termofísico, en tanto que esta cuantifica datos estadísticos independientemente de su valor epistémico (es decir, sobre si nos aportan conocimiento fáctico acerca del sistema físico en cuestión). Recordemos 
la cita de Stonier que vimos en Sección 1. Sin embargo, la pretensión brillouiniana de formalizar la noción cotidiana en el contexto teórico de la mecánica estadística sufre a su vez de ciertas desventajas frente al concepto shannoniano, entre las más importantes encontramos la extrema dificultad a la hora de cuantificar estados epistémicos termofísicamente relevantes "the everyday notion of information does not really admit the kind of precise degree of weighting of amount that a quantitative measure (such as $\mathrm{H}(X)$ ) would import" (Timpson 2013, p.30). Será precisamente la pretensión de identificar esta medida con el concepto técnico de Shannon (tal y como analizaremos en Sección 5.2) en la descripción de un proceso como el visto en Figura 1 lo que provoca la emergencia de la paradoja info-térmica.

\subsection{Interpretaciones informacionales de la entropía mecánico estadística}

Otra de las formas en la que dicha paradoja puede surgir dentro de una descripción informacionalista es mediante el uso de ciertas interpretaciones sub-definidas y teóricamente ilegítimas (más o menos entrelazadas mutuamente) de los conceptos infoentrópicos empleados, principalmente la interpretación de la entropía como 'falta de información' o la interpretación de la entropía como una noción información-teórica. Procedemos a analizar ambas a continuación.

Tal y como señalamos en la Sección 2.2, el principio de Neguentropía (estableciendo una relación recíproca entre información y entropía) sobre el que se fundamenta el paradigma brillouiniano parte en cierto modo de la interpretación del concepto físico de entropía como 'falta de información' o más propiamente como 'falta de información acerca de la configuración microscópica de un sistema'. Dicha interpretación aparece por primera vez en una famosa cita del químico ganador del Premio Nobel Gilbert Lewis a principio de los años treinta:

Gain in entropy always means loss of information, and nothing more. It is a subjective concept, but we can express it in a least subjective form as follows. If on a page we read the description of a physic chemical system, together with certain data which help to specify the system, the entropy of the system is determined by those specifications. If any of the essential data are erased, the entropy become less. (Lewis 1930)

El lector advertirá que la noción de información empleada por Lewis en esta cita es la usual o cotidiana, de evidentes connotaciones epistémicas y semánticas (información-conocimiento acerca de un sistema físico-químico), puesto que además la única definición cuantitativa de información disponible en esa época aparece de manos de Richard Hartley (1929) apenas un años antes. ${ }^{9}$ Además, la propuesta interpretativa de Lewis contiene por primera vez la relación de reciprocidad entre la entropía de un sistema e información sobre un sistema, que será utilizada posteriormente por 
Brillouin. Lo que nos concierne directamente a la temática de este artículo es que, si nos detenemos a analizar esta decisiva cita en profundidad, nos encontraremos con la primera muestra histórica de la paradoja info-térmica: por un lado, si la entropía significa falta de información (interpretación lewisiana) entonces esta segunda disminuirá si la primera aumenta; por otra parte y como sugiere al final de la cita, si la entropía de un sistema decrece, los 'datos esenciales' para especificar estadísticamente el sistema decrecen a su vez. Podemos interpretar caritativamente el contenido de los 'datos esenciales' a los que Lewis hace referencia como una suerte de descripción estadística proto-shannoniana codificada en la distribución de posiciones y velocidades. No es ni mucho menos baladí el hecho científico-histórico de que la primera conexión explícita entre entropía e información contenga en su seno la paradoja infotérmica.

Otra de las interpretaciones más problemáticas que encontramos en el panorama informacionalista es la llevada a cabo por Warren Weaver, coautor de la teoría matemática de la comunicación junto con Shannon, el cuál (partiendo de ciertas ideas de von Neumann y de la interpretación lewisiana de entropía) sugiere que la medida de entropía boltzmanniana constituye un concepto intrínsecamente informaciónteórico:

Dr. Shannon's work roots back, as von Neumann has pointed out, to Boltzmann's observation, in some of his work on statistical physics (1894), that entropy is related to "missing information," inasmuch as it is related to the number of alternatives which remain possible to a physical system after all the macroscopically observable information concerning it has been recorded. (Shannon \& Weaver 1949, p.3)

La justificación de esta interpretación es que los macroestados de Boltzmann (o complexiones de Plank) pueden entenderse información-teóricamente: por cada bit o unidad binaria de información que se tiene acerca del microestado molecular del sistema, el volumen fásico del macroestado que describe estadísticamente al sistema cinético se reduce a la mitad. Es decir, por cada bit de información microscópica que obtiene un observador se eliminan la mitad de microestados macroscópicamente indistinguibles. En este sentido, la cantidad de bits necesarios para especificar el microestado del sistema viene determinado por el volumen del macroestado que describe el sistema; lo cual quiere decir que para determinar la configuración microscópica de un gas ideal con una notable resolución serían necesarios al menos $10^{23}$ bits de información (cantidad superior a la contenida en todos los dispositivos de almacenamiento del planeta). Sin embargo, cambiar la base logarítmica de la medida entrópica de Boltzmann no reporta ningún beneficio (i) formal, en tanto que la codificación en 'nats' es más eficiente dentro del ámbito de las ciencias físicas, (ii) teórico, dado que no produce mejores modelos de fenómenos físicos, o (iii) epistémico, puesto que no 
explica ni predice nada más allá de la mecánica estadística estándar. Por tanto, esta no solo es una interpretación anacrónica (otorga carácter información-teórico) sino también científicamente trivial. Como se puede notar, esta estrategia weaveriana conecta interpretativamente el formalismo mecánico estadístico de Boltzmann (parte brillouiniana de la paradoja) con el mecanismo bit-codificador información-teórico de Shannon (parte jaynesiana de la paradoja), nutriendo el terreno para que florezca nuestra paradoja.

$$
* * *
$$

Resumamos lo visto en esta sección. Por una parte, las carencias semánticas del concepto de Shannon provocan graves deficiencias epistémicas a la hora de aplicarlo en contextos termofísicos, por ello o se desarrolla un concepto alternativo para el mismo objetivo científico (à la Brillouin) o bien se lleva a cabo una 'semantificación' del mismo. Sin embargo, la información brillouiniana sufre a su vez la desventaja de ser incapaz de cuantificar rigurosamente los estados informacionales de un observador con respecto a los sistemas termo-cinéticos, por lo que este concepto podría mejorarse mediante una 'tecnificación' suficientemente sofisticada del mismo. Entre los modos de dar significación termofísica a los diferentes conceptos de información (incluyendo la noción ordinaria) también encontramos las interpretaciones informales de la entropía como medida de la 'falta de información' o como cantidad intrínsecamente información-teórica. Todas estas estrategias conceptuales y caminos interpretativos, explorados de un modo otro por las diferentes tendencias informacionalistas pasadas y presentes, dan lugar a nuestra paradoja (Figura 2), como mostramos a continuación.

\section{Casos de estudio de la paradoja}

Acabamos de analizar los complejos entramados conceptuales e interpretativos que dan lugar a la paradoja infotérmica que aquí nos incumbe. De acuerdo con lo visto previamente, el contenido de esta no proviene de sostener simultáneamente el principio brillouiniano de Neguentropía y el jaynesiano de Máxima Entropía (aunque esto sucede habitualmente en el informacionalismo contemporáneo), sino que se explicaría a partir de un cúmulo de concepciones inconsistentes e interpretaciones injustificadas desarrolladas a lo largo de la evolución histórica del informacionalismo en las ciencias físicas. Ahora procederemos a ver como nuestra paradoja surge de acuerdo a las causas conceptual-interpretativas en dos casos concretos de análisis informacional de procesos térmicos. 


\section{Paradoja Info-Térmica}

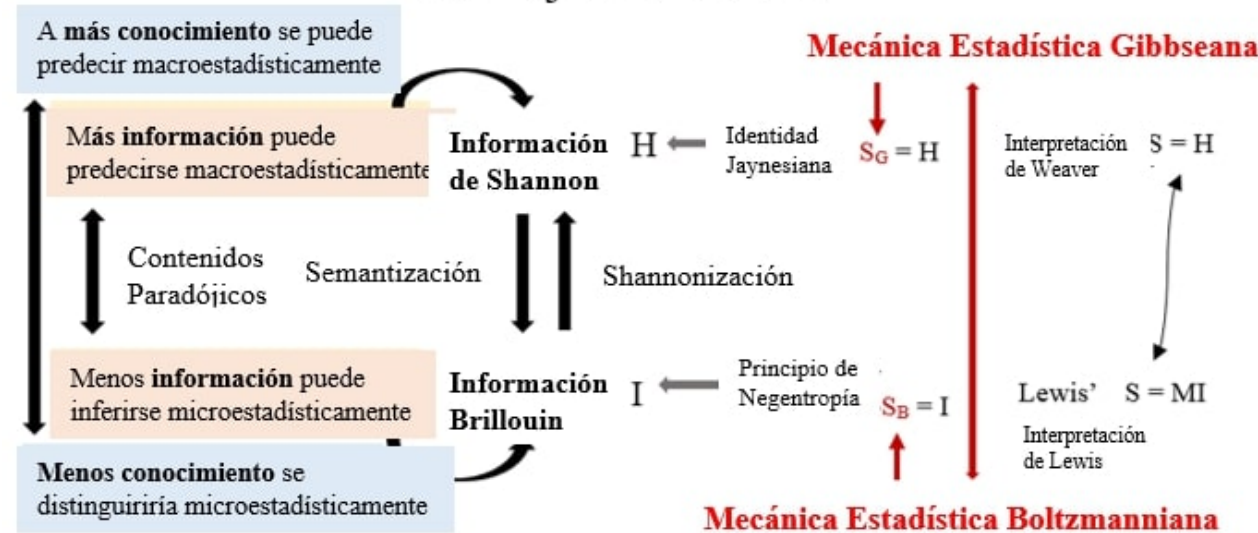

Figura 2: Esquema conceptual de la paradoja info-térmica. Se muestra diagramáticamente como las descripciones jaynesianas (parte superior del esquema) y las brillouinianas (parte inferior) se fundan teóricamente en la mecánica estadística gibbseana y boltzmanniana, respectivamente. Estas además se conectan a través de (a) la semantización de la información de Shannon, (b) la 'tecnificación' de la información brillouiniana o (c) la adopción de interpretaciones injustificadas (como las desarrolladas por Lewis o Weaver), de cuyas inconsistencias conceptuales e incoherencias interpretativas se deriva el contenido paradójico de la tesis infotérmica.

\subsection{Brillouin y la identificación de entropía e información semántica}

En el propio Brillouin, protagonista de gran parte de nuestro análisis como hemos visto, encontramos un ejemplo cristalino de como la 'semantificación' de la noción informacional de Shannon puede dar lugar a la paradoja info-térmica. En la sección "Problems of Semantic Information" (1962, Chapter 20, Section 6), el físico francoalemán esbozó una estrategia argumental (completamente inadvertida para la literatura posterior) para identificar su medida neguentrópica no solo con la noción ordinaria de información (tal y como hemos venido defendiendo hasta ahora) sino también con ciertas nociones técnicas de información semántica, especialmente la definida por Carnap y Bar-Hillel (1952) una década antes. Cabe destacar que uno de los pasos argumentales implícitos en esta estrategia es el de identificar conceptualmente su noción de información I (ratios de macroestados boltzmannianos o complexiones de Plank) con la medida informacional de Shannon $\mathrm{H}$, insatisfactoriamente justificado a lo largo de su opera magna Science and Information Theory (1962). La razón principal de este fracaso teórico es que Brillouin únicamente pretendió salvar las diferencias 
formales que existen entre ambas cantidades (base logarítmica, signo de la expresión matemática) y no adaptar robustamente los mecanismos representacionales de $\mathrm{H}$ al ámbito teórico físico:

The connection between entropy and information were discovered by Shannon, but he defined entropy with a sign opposite to that of the standard thermodynamical definition. Hence what Shannon calls entropy of information actually represents neguentropy. (Brillouin 1962, p.166)

La medida de información semántica 'inf(i)' de Carnap y Bar-Hillel fue desarrollada como un concepto formal que solventaba la insensibilidad semántica de la medida de Shannon a partir de los recursos teóricos de la lógica inductiva. Brillouin explicitó que, por definición, la cantidad de información semántica 'inf(i)', definida como el número de estados equiprobables que se reducen a partir del contenido de la proposición $i$, es equivalente a $\log _{2}\left(\mathrm{~N}_{\mathrm{r}} / \mathrm{N}\right)$ o como la cantidad de bits requeridos para especificar un estado microscópico $x$ tras comprimir/expandir el espacio de estados macroscópicamente compatibles $X$. Como cabría esperar, además de ser una definición formal clásica de información semántica defectuosa en muchos sentidos, la medida cuantitativa de información de Bar-Hillel-Carnap no puede contener significado termo-físico por múltiples motivos: el fundamental es su incapacidad de desarrollar modelos físicos significativos para la práctica científica.

Retomemos en este nuevo escenario teórico el modelo cinético concreto de una expansión irreversible, tal y como queda ilustrado en Figura 1, para evaluar la consistencia de sus conceptos. Asumamos por otra parte que el sistema gaseoso se encuentra en su extremadamente improbable estado inicial (moléculas dispuestas regularmente en una parte del contenedor) y mediante una inferencia experimental obtenemos la proposición $i=$ "todas las moléculas del gas se encuentran posicionadas en la cuarta parte del volumen a la izquierda", con lo que el macroestado representando nuestro conocimiento estadístico del gas se reduce logarítmicamente. En esta situación particular se incrementa la información brillouiniana-carnapiana (de evidente carácter semántico-epistémico) de un observador acerca del gas por medio de la proposición $i$ debido a la reducción de complexiones microscópicas indistinguibles; simultáneamente, la cantidad de bits requeridos para determinar (o 'codificados en', si uno adopta una posición óntica) la disposición de moléculas en el envase también disminuye considerablemente. De nuevo, aquí nos encontramos con la paradoja info-térmica.

\subsection{La aproximación informacionalista a la física térmica de Ben-Naim}

Uno de los casos más evidentes dentro de la literatura informacional actual en los que acontecen la mayor parte de los conceptos-interpretaciones expuestas en la sección anterior (generando un contexto propicio para la paradoja info-térmica) es en 
la obra científica del físico neo-jaynesiano Arie Ben-Naim (2008). En su texto $A F a$ rewell to Entropy: Statistical Thermodynamics Based on Information defiende que la noción estadístico mecánica de entropía ha de eliminarse en favor del concepto de 'falta de información' (MI), el cual se define mediante la misma expresión matemática que la entropía (salvo por la constante de Boltzmann ' $k$ ') y da cuerpo formal a la tesis interpretativa de Lewis (1930), tal y como expusimos en Sección 4.2. Nótese que el autor acepta tanto la interpretación lewisiana de la entropía 'falta de información' (e incluso la cuantifica matemáticamente como 'In W') como la estrategia interpretativa de Weaver al asumir el carácter información-teórico de las cantidades de carencia de información 'In W'. Sin embargo, la principal estrategia (y clave de su profunda incoherencia conceptual) de Ben-Naim es la de justificar una identificación no solo forma y cuantitativa, sino también conceptual y teórica entre su noción lewisiana semántico-epistémica de 'falta de información' y la medida informacional sub-semántica de Shannon, tal y como acabamos de ver en el caso de Brillouin (Sección 5.1). En este sentido defiende explícitamente que "the measure of 'information' as defined by Shannon also retains some of the flavor of the meaning of information as we use in everyday life" (p.17)

Uno de los tantos modelos moleculares usados por Ben-Naim (2008, p.265-268) en los que emerge la paradoja info-termal de un modo más evidente es el de un proceso mecánico estadístico de mezcla de dos gases combinado con una expansión irreversible, fenómeno popularizado por Gibbs (1902) dentro de la literatura termofísica para ilustrar su famosa paradoja. Imaginemos que inicialmente tenemos un contenedor dividido por una membrana en dos volúmenes iguales $\mathrm{V}$ ambos repletos de gases compuestos de moléculas $\mathrm{N}_{\mathrm{A}}$ y $\mathrm{N}_{\mathrm{B}}$ (respectivamente); al eliminar la membrana, el gas ahora conformado por $\mathrm{N}_{\mathrm{A}} \mathrm{N}_{\mathrm{B}}$ pasará a ocupar un volumen $2 \mathrm{~V}$. En el caso en el que los dos gases sean químicamente idénticos $(A=B)$ no se generará ninguna cantidad de entropía (ni por tanto cambiará la falta de información, en el lenguaje de Ben-Naim); sin embargo, cuando dichos gases distintos $(A \neq B)$ la cantidad de falta de información aumentará en $\left(\mathrm{N}_{\mathrm{A}}+\mathrm{N}_{\mathrm{B}}\right)$ In2. De acuerdo con las palabras del autor neo-jaynesiano, la falta de información o 'MI' aumenta, o dicho de un modo más simple, la información brillouiana sobre (remarcando su carácter semántico-epistémico) el microestado actual disminuye, en tanto que nuestra capacidad de inferir la configuración molecular actual decrece debido a que el número de configuraciones macroscópicamente indistinguibles se dobla al final del proceso. Por esta misma razón y en tanto que acepta la interpretación Weaveriana (ver Sección 4.2), la cantidad de información de Shannon también se duplica durante el proceso puesto que necesitaremos el doble de bits para especificar el microestado actual del gas. Esta descripción contiene precisamente la paradoja info-térmica: mientras la información sobre la configuración actual del gas disminuye al final del proceso, la información codificada en estructuras macroscópicamente indistinguibles incrementa 
de manera simultánea.

La tesis que en este punto quiero defender es que la aparición de nuestra paradoja durante el análisis informacional à la Ben-Naim de un proceso de mezcla-expansión es una muestra robusta del hecho de que dicha aproximación informacional a la física térmica (así como posiciones relacionadas) se asienta sobre bases conceptual e interpretativamente inconsistentes. En consecuencia, dichas aproximaciones informacionales conceptualmente deficientes resultarán epistémicamente poco fructíferas, en términos de comprensibilidad de fenómenos, explicaciones inválidas o predicciones incorrectas. Muestra de ello es que la propuesta información-céntrica de Ben-Naim no solo no soluciona la conocida como 'paradoja de Gibbs', en la que considerar la entropía como una cantidad intensiva (apelando a una indistinguibilidad microscópica de partículas) conlleva aparentes violaciones de la segunda ley de la termodinámica, sino que además genera paradojas adicionales como la que aquí nos concierne. Además, a partir del concepto de 'falta de información' es imposible explicar (tal y como reconoce el propio Ben-Naim [2008, p.276]) el incremento de entropía termodinámica en un proceso de expansión y mezcla de diferentes partículas sino apelando a propiedades intensivas ad hoc de dichas partículas. Esta explicación es por supuesto invalida, ya que la causa propiamente termodinámica del incremento de entropía durante este proceso es el incremento de volumen del recipiente en el que se encuentra el gas. Estas y otras deficiencias epistémicas de la aproximación informacional de Ben-Naim (como uno de los principales representantes actuales del informacionalismo termofísico) muestran como la paradoja info-térmica, analizada en profundidad a lo largo de este artículo, no es sino el síntoma de una falta de rigor conceptual

\section{Conclusiones}

A lo largo de este artículo hemos llevado a cabo un análisis meticuloso de la aquí denominada 'paradoja info-térmica', la cual establece (en términos generales) que cuando un modelo cinético-molecular o gas se aproxima a su equilibrio térmico la información del observador incrementa y disminuye simultáneamente. Tal y como se ha argumentado, la paradoja surge (entre otras razones) a partir de la confluencia histórico-científica de dos de los principales paradigmas informacionales dentro de las ciencias físicas: a saber, el jaynesianismo (Jaynes 1957), basado fundamentalmente en la identificación conceptual de la entropía estadístico mecánica y la medida informacional de Shannon, y el brillouinismo (Brillouin 1962), centrado en el 'principio de neguentropía' que iguala información (semántica, acerca de la conformación molecular de un sistema) y entropía negativa. A partir de estos elementos podemos distinguir las dos tesis que componen la paradoja info-térmica: por un lado, (a) que la información técnica Shannoniana (determinable a partir de la identificación jay- 
nesiana) de un modelo cinético incrementa logarítmicamente en su aproximación al equilibrio térmico; por otra parte (b) que la información semántico-epistémica brillouiana (especificable mediante el principio de neguentropía) sobre el estado microscópico del sistema disminuye de manera simultánea. Cabe destacar que cualquier distinción entre diferentes conceptos (shannoniano y brillouiano-ordinario), tipos de información física (macroscópico-estadística y microscópico-determinista) u otras distinciones dependientes de modelos particulares no elimina el carácter paradójico de nuestra tesis. En esta cita de Charles Seife, adalid del informacionalismo físico actual, se muestra claramente esta pretensión disolutiva:

Sometimes you will hear people say entropy is the same thing as information; sometimes you will hear people say that information is negative entropy or negentropy. The difference arises because people are accustomed to analyzing different things. Some are looking at the sender and the unpredictability of a potential message, and some are looking at the receiver and the uncertainties about the answer to a question. In truth, both are looking at the same thing: sender and receiver are two sides of the same coin. (Seife 2006 p.273)

En contra de lo que defiende Seife, ni apelar al modelo comunicativo emisor-receptor ni afirmar que la predictibilidad macroscópica y la incertidumbre microscópica sean fenómenos epistémicos diferentes resuelven la paradoja. En este artículo hemos propuesto una explicación a diferentes niveles del origen causal de la paradoja: (a) se busca dotar de contenido semántico a la medida informacional de Shannon ' $\mathrm{H}$ ' (asumiendo que esta codifica información sobre el estado microscópico actual) dentro del formalismo mecánico estadístico (Sección 4.1 y 5.1), (b) se pretende cuantificarformalizar rigurosamente información semántica como la brillouiana 'I' al estilo noción estadística de Shannon (Sección 4.1. y 5.2); y por último, (c) se llevan a cabo interpretaciones informacionales infundadas de las medidas entrópicas, como la de Lewis o la de Weaver (Sección 4.2). De este modo, el contenido de ambas partes de la tesis se mantiene conceptual-interpretativamente indisociable y (lo que es más importante) paradójicamente conectadas: ya sea mediante inferencias estadísticas o a través de la discriminación de configuraciones indistinguibles, el objetivo común es la adquisición de 'información' (en su acepción cotidiana) o 'conocimiento' relativo al comportamiento termodinámico de ciertos sistemas en función de sus componentes moleculares.

Una de las principales ideas que he defendido a lo largo de este artículo es que la paradoja info-térmica no es sino la consecuencia inmediata de la inconsistencia conceptual e interpretativa subyacente a las aproximaciones informacionales a la termofísica, tal y como hemos visto para el caso clásico de Brillouin (1962), padre fundador del informacionalismo, o el ejemplo de Ben-Naim (2008), uno de las mayores representantes del informacionalismo actual. Lo cierto es que esta profunda deficiencia 
conceptual de la física informacional no es inerte en cuanto al funcionamiento de dicha teoría. Por el contrario, el uso de nociones y aparatos informacionales formales no supone ninguna ventaja epistémica añadida a la hora de confirmar, comprehender, explicar o predecir fenómenos térmicos con respecto al modo en el que la termodinámica o la mecánica estadística lleva a cabo dichas tareas científicas. Dejaré la defensa particular de dicha tesis para un futuro artículo.

\section{Referencias}

Albert, D. Z. 2000. Time and Chance. Cambridge, Mass.: Harvard University Press.

Ben-Naim, A. 2008. A Farewell to Entropy. Statistical Thermodynamics Based on Information. World Scientific.

Bennett, C. H. (1982) The Thermodynamics of Computation - a Review, International Journal of Theoretical Physics 21: 905-940. Reprinted in Leff and Rex (1990), pp.213-248.

Boltzmann, L. 1894. On the application of the determinantal relation to the kinetic theory of gases. Reprinted in Boltzmann 1909, Vol. III, pp.520--5.

Brillouin, L. 1951. Maxwell's Demon Cannot Operate: Information and Entropy. I, Journal of Applied Physics 22: 334-337. Reprinted in Leff and Rex (1990), pp. 134-137. Maxwell's Demon Cannot Operate: Information and Entropy. II, Journal of Applied Physics 22: 338343.

Brillouin, L. 1953. The Negentropy Principle of Information, Journal of Applied Physics 24: 1152-1163.

Brillouin, L. 1962. Science and Information Theory. New York: Academic Press.

Callender, C. 1999. Reducing thermodynamics to statistical mechanics: The case of entropy. Journal of Philosophy 96 (7): 348-373.

Carnap, R. \& Bar-Hillel, Y. 1952. An Outline of a Theory of Semantic Information. Technical Report No. 247, Research Laboratory of Electronics, MIT.

Davies, P \& Gregersen, N. H. 2010. Information and the Nature of Reality. From Physics to Metaphysics. Cambridge: Cambridge University Press.

Denbigh, K. G. 1981. How Subjective is Entropy? Chemistry in Britain 17: 168-185. Reprinted in Leff and Rex (1990), pp.109-115.

Denbigh, K. G. \& Denbigh, J. S. 1985. Entropy in Relation to Incomplete Knowledge. Cambridge: Cambridge University Press.

Earman, J. \& Norton, J. 1998. Exorcist XIV: the wrath of Maxwell's demon. Part I. From Maxwell to Szilard. Studies in History and Philosophy of Modern Physics 29(4): 435-471.

Earman, J. \& Norton, J. 1999. Exorcist XIV: the wrath of Maxwell's Demon. Part II. From Szilard to Landauer and beyond. Studies in History and Philosophy of Modern Physics 30(1): $1-40$.

Frigg, R. 2004. In what sense is the Kolmogorov-Sinai entropy a measure for chaotic behaviour? Bridging the gap between dynamical systems theory and communication theory. British Journal for the Philosophy of Science 55: 411-34.

Frigg, R. \& Werndl, C. 2011. Entropy - A Guide for the Perplexed. In Claus Beisbart \& Stephan Hartmann (eds.), Probabilities in Physics. Oxford University Press, pp.115-142. 
Gibbs, J. W. 1902. Elementary Principles in Statistical Mechanics: Developed with Especial Reference to the Rational Foundation of Thermodynamics. New Haven, Conn.: Yale University Press. Reprinted Mineola, N.Y.: Dover, 1960, and Woodbridge, Conn.: Ox Bow Press, 1981.

Hartley, R. 1928. Transmission of information. Bell System Technical Journal 7: 535-63.

Hilgevoord, Jan \& Uffink, Jos 1991. Uncertainty in prediction and in inference. Foundations of Physics 21(3): 323-341.

Jaynes, E. T. 1957a. Information theory and statistical mechanics, Physics Review 106: 620630.

Jaynes, E. T. 1957b. Information theory and statistical mechanics. II, Physics Review 108: 171-190.

Landauer, R. 1961. Irreversibility and Heat Generation in the Computing Process, IBM Journal of Research and Development 5: 183-91. Reprinted in Leff and Rex (1990), pp.188-196.

Landauer, R. 1998. Information is Inevitably Physical. Submitted to Feynman Lectures on Computation, vol.2, edited by A. J. G. Hey (Addison Wesley, Reading).

Leff, H. S. \& Rex, A. F. 1990. Maxwell's Demon: Entropy, Information, Computing. Princeton: Princeton University Press.

Leff, H. S. \& Rex, A. F. 2003. Maxwell's Demon 2: Entropy, Classical and Quantum Information, Computing. Philadelphia, Pennsylvania: Institute of Physics Publishing.

Lewis, G. N. 1930. The symmetry of time in physics, Science 71: 569-577.

Lloyd, S. 2006. Programming the Universe: A Quantum Computer Scientist Takes On the Cosmos. New York: Alfred A. Knopf.

Marcos A. 2011. Bioinformation as a Triadic relation. In: G. Terzis \& R. Arp (eds.), Information and Living Systems: Philosophical and Scientific Perspectives, M.I.T. Press, pp. 55-90).

Maxwell, J. C. 1867. Letter to P.G. Tait, 11 December 1867. Life and Scientific Work of Peter Guthrie Tait, C. G. Knott (author), Cambridge: Cambridge University Press, 1911, pp.213215.

Maxwell, J. C. 1952. Scientific Papers of James Clark Maxwell, edited by W. D. Niven, New York: Dover.

Norton, J. 2005. Eaters of the lotus: Landauer's principle and the return of Maxwell's demon. Studies in the History and Philosophy of Modern Physics 36: 375--411.

Parker, D. 2011. Information-Theoretic Statistical Mechanics without Landauer's Principle. British Journal for the Philosophy of Science 62(4): 831-856.

Rodd, P. 1964 Some Comments on Entropy and Information, American Journal of Physics 32: 333-335. Reprinted in Leff and Rex (1990), pp.145-147.

Rothstein, J. 1952. Information and Thermodynamics. Physics Review 85: 135.

Seife, C. 2007. Decoding the Universe: How the New Science of Information is Explaining Everything in the Cosmos from our Brains to Black Holes. Ed Penguin Group.

Schrödinger, E. 2004. What is Life? (11th reprinting ed.). Cambridge: Canto.

Shannon, C. E. 1948. A mathematical theory of communication. Bell System Technical Journal 27: 379-423, 623--56.

Shannon, C. E. \& Weaver, W. 1949. The Mathematical Theory of Communication. Urbana, Ill., Chicago, Ill. \& London: University of Illinois Press.

Sklar, L. 1993. Physics and Chance: Philosophical Issues in the Foundations of Statistical Mechanics, Cambridge: Cambridge University Press.

Stonier, T. 1990. Information And The Internal Structure Of The Universe. Springer-Verlag. 
Szilard, L. 1929. On the Decrease of Entropy in a Thermodynamic System by the Intervention of Intelligent Beings', in The Collected Works of Leo Szilard: Scientific Papers (MIT Press, 1972), pp.120-129. Reprinted in Leff and Rex (1990), pp.124-133.

Szilard, L. 1978. The Collected Works of Leo Szilard; Scientific Papers, Boston, MA: MIT Press. Timpson, C. G. 2013. Quantum Information Theory and the Foundations of Quantum Mechanics. Oxford University Press.

Thims, L. 2012. Thermodynamics $\neq$ Information Theory. Journal of Human Thermodynamics 8(1): 1-120.

Thomson, W. 1853. On the Dynamical Theory of Heat, with numerical results deduced from Mr. Joule's equivalent of a Thermal Unit, and M. Regnault's Observations on Steam. Philosophical Magazine Ser. 4, VI: 8-21.

Tribus, M. \& McIrvine, E. C. 1971. Energy and information. Sci. Am. 225: 179-188.

Vedral, V. 2010. Decoding Reality: The Universe as Quantum Information. Oxford University Press.

Von Helmholtz, H. 1882. Wissenschaftliche Abhandlungen, Three volumes: second volume 1883, third volume 1895. Leipzig: Johann Ambrosius Barth.

Von Neumann, J. 1948. The general and logical theory of automata. In Cerebral Mechanisms in Behavior: The Hixon Symposium, pp. 1-31.

Von Neumann, J. 1955. Mathematical Foundations of Quantum Mechanics. Princeton: Princeton University Press, published originally in German in 1932, Ch. V.

Wiener, N. 1948. Cybernetics: Or Control and Communication in the Animal and the Machine. Paris: Hermann \& Cie \& Cambridge: MIT Press.

\section{Notas}

${ }^{1}$ Siguiendo a Parker (2011) podríamos además distinguir así mismo un tercer paradigma basado en el análisis de los costes físicos de ciertos procesos informacionales como la adquisición o eliminación de información (Brillouin 1962, Landauer 1961). Dicho paradigma no será tratado en este artículo (a pesar de su enorme impacto en la literatura físicoinformacionalista) en tanto que no posee relevancia alguna para el tema que aquí nos concierne.

${ }^{2}$ Además, las funciones logarítmicas contenidas en la entropía de Shannon suelen codificarse en unidades binarias o 'bits', mientras que la entropía de Gibbs se mide mediante logaritmos en base diez, cuantificando en unidades naturales o 'nats'.

${ }^{3}$ Podemos encontrar algunos autores, como por ejemplo Denbigh (1981), que criticarán esta estrategia conceptual como una mera adopción terminológica que no refiere sino a cambios entrópicos de un sistema. De este modo, la interconvertibilidad entre neguentropía e información presupuesta por el principio neguentrópico brillouiniano se presentaría no como una ley natural (necesaria) sino como una tesis trivial sin ningún valor explicativo.

${ }^{4}$ Entre los autores claves en este proceso de sincretismo informacional nos encontramos al físico y economista Myron Tribus (Tribus \& McIrvine [1971]), un discípulo intelectual de Brillouin que acabó convirtiéndose también al jaynesianismo, integrando diversos elementos de ambas tradiciones en su obra científico-divulgativa principalmente durante los años setenta. 
${ }^{5}$ En este paper, Frigg identifica la entropía informacional de Shannon con la entropía de Kolmogorov-Sinai, interpretando ambas como medidas de la impredecibilidad de un sistema física.

6 Algunos autores neo-dretskeanos, como recientemente Martínez (2018) o Alastair (2019), defienden que bajo el concepto informacional shannoniano subyace una cierta semántica informacional, desplegada mediante mecanismos interpretativos o ciertos añadidos formales como puntos 'dulces' en ratios de distorsión o correlaciones estadísticas en ratios logarítmicos.

${ }^{7}$ Agradezco a un revisor anónimo el haberme sugerido que clarificase esta cuestión.

${ }^{8}$ Timpson remarca "We could accept that quantity [Shannon information $\mathrm{H}$ ] as sometimes measuring an amount of information in the everyday sense, but only sometimes. That is, when the scenario is paired down to the extent that one's only interest is the given experiment and what the probabilities of its various outcomes are: a situation when none of the other standard features involving what one learns, or what one be might infer, or what one's various interest might be, are in play. (...) But one will note that this is far from a representative epistemic scenario" (Timpson 2013, p.30)

${ }^{9}$ La medida informacional de Hartley $(c \log n$ ) cuantifica la cantidad de información contenida en $n$ número de alternativas con igual probabilidad de ocurrencia.

\section{Agradecimientos}

I should particularly thank Carl Hoefer for his useful comments. This work has been funded by a FPU Grant (FPU16/0774) of the Spanish Ministry of Education. It has been developed under the research framework of LOGOS and the research group "Laws, explanation and realism in physical and biomedical sciences" (FFI2016-76799-P). 